\title{
Shear and bulk viscosities of the gluon plasma in a quasiparticle description
}

\author{
M. Bluhm, ${ }^{1,2}$ B. Kämpfer, ${ }^{3,4}$ and K. Redlich ${ }^{2,5}$ \\ ${ }^{1}$ SUBATECH, UMR 6457, Université de Nantes, Ecole des Mines de Nantes, IN2P3/CNRS, 4 rue Alfred Kastler, \\ FR-44307 Nantes cedex 3, France \\ ${ }^{2}$ CERN, Physics Department, Theory Division, CH-1211 Geneva 23, Switzerland \\ ${ }^{3}$ Institut für Strahlenphysik, Helmholtzzentrum Dresden Rossendorf, PF 510119, DE-01314 Dresden, Germany \\ ${ }^{4}$ Institut für Theoretische Physik, TU Dresden, DE-01062 Dresden, Germany \\ ${ }^{5}$ Institute of Theoretical Physics, University of Wroclaw, PL-50204 Wroclaw, Poland
}

(Received 29 November 2010; revised manuscript received 2 February 2011; published 8 August 2011)

\begin{abstract}
Shear and bulk viscosities of deconfined gluonic matter are investigated within an effective kinetic theory by describing the strongly interacting medium phenomenologically in terms of quasiparticle excitations with medium-dependent self-energies. We show that the resulting transport coefficients reproduce the parametric dependencies on temperature and coupling obtained in perturbative QCD at large temperatures and small running coupling. The extrapolation into the nonperturbative regime results in a decreasing specific shear viscosity with decreasing temperature, exhibiting a minimum in the vicinity of the deconfinement transition, while the specific bulk viscosity is sizable in this region, falling off rapidly with increasing temperature. The temperature dependence of specific shear and bulk viscosities found within this quasiparticle description of the pure gluon plasma is in agreement with available lattice QCD results.
\end{abstract}

DOI: 10.1103/PhysRevC.84.025201

PACS number(s): 12.38.Mh, 25.75.-q, 52.25.Fi

\section{INTRODUCTION}

Transport properties of strongly interacting matter, encoded in coefficients such as shear and bulk viscosities, which describe the hydrodynamic response of the system to energy and momentum density fluctuations, are of particular importance for understanding the nature of QCD matter. Their firm knowledge, aside from other characteristics such as the QCD equation of state (EoS), has great impact on a variety of physical phenomena in cosmology, astrophysics, or nuclear physics. In particular, the dynamical description of the medium created in relativistic heavy-ion-collision experiments requires a precise determination of the temperature $T$ and density dependencies of these transport coefficients. Even though tremendous efforts have been invested both from theoretical as well as experimental sides, this remains still a challenging task, especially in the regime of experimentally accessible temperatures and densities.

It was shown that the observed collective flow behavior of the matter created at the Relativistic Heavy Ion Collider (RHIC), in particular, the measured elliptic flow [1-4], could quantitatively be described by means of ideal hydrodynamics [5-10] for not too large transverse momenta of the particles. Although the results of these calculations depend crucially on the employed freeze-out prescription as well as the assumed initial conditions [11-18], the success of ideal hydrodynamics suggested, at most, small dissipative effects in the medium produced at RHIC [19-21]. This observation led to the interpretation that the produced quark-gluon plasma (QGP) behaves as a strongly coupled fluid rather than a weakly interacting gas [22-24]. Detailed calculations within the framework of relativistic dissipative fluid dynamics were performed [13,25-38], confirming indeed the smallness of dissipative effects. Therein, the equations of (for many EoS) causal Israel-Stewart theory [39,40] are solved. Recently, however, corrections to this second order in gradients theory were reported [41-43].

On the other hand, some transport coefficients, in particular the shear viscosity $\eta$, can not be arbitrarily small. In fact, the quantum-mechanical uncertainty relation sets a fundamental lower bound on $\eta$ for any physical system [44]. Based on unitarity arguments [45-47], the dimensionless specific shear viscosity (i.e., the ratio of shear viscosity to entropy density $s$ ) was recently conjectured to be bound from below by $(\eta / s)_{\mathrm{KSS}} \geqslant \hbar /(4 \pi)$ (Kovtun-Son-Starinets bound). The comparison of causal viscous hydrodynamic calculations with data on low-momentum elliptic flow spectra measured at RHIC showed that $\eta / s$ should be close to this lower bound $[13,20,21,31,33,48]$. Nevertheless, some other results seem to indicate that the KSS bound might be violated for the QGP at RHIC [20,31,36]. Moreover, there are other physical systems [49-52] where this is indeed the case.

The specific shear viscosity $\eta / s$ as a function of temperature is known to exhibit a minimum in the vicinity of a phase transition for a variety of liquids and gases [47,53] as well as for ultracold fermionic systems close to the unitarity limit [54]. This gave rise to the conjecture $[20,53]$ that $\eta / s$ for QCD matter exhibits a similar behavior in the vicinity of the deconfinement phase transformation.

In contrast, the bulk viscosity $\zeta$ is exactly zero for conformally invariant systems. This is due to the vanishing trace of the energy-momentum tensor in conformal theories. In QCD, as quantum fluctuations break the scale invariance, the bulk viscosity is nonzero at finite $T$ and approaches zero only for asymptotically large $T$. In the vicinity of the deconfinement phase transformation, the dimensionless specific bulk viscosity $\zeta / s$ is expected to become large and even to diverge at a second-order phase transition [55-61]. However, there are 
examples of strongly coupled theories [62,63] for which the contrary is found.

Transport coefficients of strongly interacting systems have been calculated in a variety of different approaches. Despite the fact that lattice QCD studies of dynamical quantities, such as viscosities, are tedious to perform, they represent the first-principles nonperturbative method in the strong-coupling regime. Recently, first results for shear and bulk viscosities obtained within lattice QCD simulations were reported in [64-67].

In quantum field theories, calculations of transport coefficients are often performed within the framework of linear response theory resulting in the known Kubo formulas [68-70]. This approach for determining shear and bulk viscosities was exploited for a weakly coupled scalar field theory in $[71,72]$. Likewise, kinetic theory can be applied as a rigorous tool to quantify transport coefficients at weak coupling from a linearized Boltzmann equation. In fact, it was shown for weakly coupled scalar fields [71,72] and for hot QED [73,74] that both approaches yield equivalent results when a linearized Boltzmann equation for excitations with temperature-dependent self-energies and scattering amplitudes is considered. In QCD, kinetic-theory weak-coupling results were reported for the shear viscosity at leading logarithmic order [75] and at full leading order [76] in the QCD running coupling $\alpha_{s}$. These calculations found for $\alpha_{s} \lesssim 0.25$ a specific shear viscosity $\eta / s>1$. For the bulk viscosity perturbative QCD (pQCD), leading-order results were presented in [77].

Further investigations of transport coefficients utilize, e.g., a specific ansatz for the spectral function entering the Kubo expression for $\eta$ [78-80] or apply sum rules for the spectral density of the trace of the energy-momentum tensor, thereby relating $\zeta$ with basic thermodynamic quantities [55-57]. Moreover, within a parton cascade model based on kinetic theory [81], a value for $\eta / s \sim 0.5$ was found [82], which is much smaller than the pQCD result $\eta / s \sim 2.7[75,76]$ at comparable coupling $\alpha_{s} \sim 0.1$. Recent similar studies report $\eta / s \sim 0.8$ [83] and $\eta / s \sim 1$ [84,85] instead.

In this paper, we study the properties of shear and bulk viscosities for the pure gluon plasma at finite temperature. The dynamics of the system is considered phenomenologically by assuming that it can be described in terms of gluonic quasiparticle excitations. In the underlying quasiparticle model (QPM), the (quasi)gluon excitations obey a mediumdependent dispersion relation, where the corresponding selfenergy depends on $T$ both explicitly but also implicitly via a phenomenological effective coupling $G^{2}(T)$ [86-89]. In a pure gluon plasma, there are no conserved charges related to internal symmetries. Thus, $T$ is the only thermal variable characterizing properties of the system. The QPM was successfully employed to quantitatively describe lattice QCD results of equilibrium thermodynamics such as the EoS and related quantities [90-94]. In the following, we apply this model to systems exhibiting small departures from thermal equilibrium by using the results of an effective kinetic-theory approach [71,72] for shear and bulk viscosities derived for a quasiparticle system in [95].

We show that this quasiparticle picture combined with kinetic theory provides a powerful method to quantify the transport properties of the gluon plasma in a broad temperature range above the critical temperature for deconfinement $T_{c}$. In particular, we show that, in the high-temperature limit, the resulting transport coefficients exhibit parametrically the dependencies on $T$ and the coupling known from pQCD. We also show that our results extrapolated into the nonperturbative regime are in agreement with available lattice QCD results.

Our paper is organized as follows. In the next section, we recall the first-order nonequilibrium corrections to the energy-momentum tensor for the (quasi)gluon plasma and discuss features of its viscosity coefficients in relaxation-time approximation as well as specify the employed quasiparticle parametrization. In Secs. III and IV, the shear and bulk viscosities are quantified and compared with available lattice QCD data. The summary of our results is found in Sec. V. Throughout this paper, we use natural units, i.e., $\hbar=c=k_{B}=1$.

\section{SHEAR AND BULK VISCOSITIES}

For charge-neutral systems and in the absence of additional conservation laws, the local conservation equations for energy and momentum in relativistic hydrodynamics as effective theory of long-wavelength modes determine the evolution of the system. Phenomenologically, the energy-momentum tensor $T^{\mu \nu}$, with equations of motion $\partial_{\nu} T^{\mu \nu}=0$, is defined as decomposition into a perfect fluid (or thermal equilibrium) part $T_{(0)}^{\mu \nu}$ and a part $T_{(1)}^{\mu \nu}$, which accounts for first-order dissipative effects [96-98]: $T^{\mu \nu}=T_{(0)}^{\mu \nu}+T_{(1)}^{\mu \nu}$ with $T_{(0)}^{\mu \nu}=$ $\epsilon u^{\mu} u^{\nu}-P \Delta^{\mu \nu}$ and $T_{(1)}^{\mu \nu}=\zeta \Delta^{\mu \nu} \partial_{\alpha} u^{\alpha}+\eta S_{\alpha \beta}^{\mu \nu} \partial^{\alpha} u^{\beta}$, where $\epsilon$ and $P$ denote energy density and thermodynamic pressure of the system, respectively; both are related through the equation of state $P=P(\epsilon)$. The projector $\Delta^{\mu \nu}=g^{\mu \nu}-u^{\mu} u^{\nu}$ with normalized four-velocity $u_{\mu} u^{\mu}=1$ and metric $g^{\mu \nu}$ is orthogonal to $u^{\mu}$; the projector $S_{\alpha \beta}^{\mu \nu}=\Delta_{\alpha}^{\mu} \Delta_{\beta}^{\nu}+\Delta_{\beta}^{\mu} \Delta_{\alpha}^{\nu}-$ $2 \Delta^{\mu \nu} \Delta_{\alpha \beta} / 3$ is also $u^{\mu}$ orthogonal. The coefficients $\eta$ and $\zeta$ entering $T_{(1)}^{\mu \nu}$ denote shear and bulk viscosities, respectively. Note that, if $T_{\mu}^{\mu}$ can be formulated solely as a function of $\epsilon$, then $\zeta=0$ is found $[99,100]$. In the way $T^{\mu \nu}$ is defined, one takes into account that, from $\partial_{\nu} T^{\mu \nu}=0$, only four out of ten independent components of $T^{\mu \nu}$ can be determined, while the others follow from expanding $T^{\mu \nu}$ around $T_{(0)}^{\mu \nu}$ in terms of small space-time gradients of the fluid four-velocity field $u^{\mu}$ [99,100]. This expansion holds up to including further gradients or higher powers of $u^{\mu}$. The Landau-Lifshitz condition $[96,97] \epsilon \equiv T^{\mu v} u_{\mu} u_{v}=T_{(0)}^{\mu v} u_{\mu} u_{v}$ provides the only uniform definition of local flow for systems without additional conserved currents [44].

\section{A. Basic definitions}

Concise expressions for the shear and bulk viscosities have been derived systematically in [95] within a quasiparticle framework. In the relaxation-time $(\tau)$ approximation, one can deduce for the (quasi)gluon plasma

$$
\eta=\frac{1}{15 T} \int \frac{d^{3} \vec{p}}{(2 \pi)^{3}} n(T)\left[1+d^{-1} n(T)\right] \frac{\tau}{E^{2}} \vec{p}^{4},
$$




$$
\begin{aligned}
\zeta= & \frac{1}{T} \int \frac{d^{3} \vec{p}}{(2 \pi)^{3}} n(T)\left[1+d^{-1} n(T)\right] \\
& \times \frac{\tau}{E^{2}}\left\{\left[E^{2}-a\right] \frac{\partial P}{\partial \epsilon}-\frac{1}{3} \vec{p}^{2}\right\}^{2},
\end{aligned}
$$

where $n(T)=d\left(e^{E / T}-1\right)^{-1}$ with $d=16$ is the equilibrium distribution function, $\partial P / \partial \epsilon$ denotes the squared speed of sound, and $a \equiv T^{2} \partial \Pi(T) / \partial T^{2}$. The excitations obey the dispersion relation $E^{2}=\vec{p}^{2}+\Pi(T)$, with $\Pi(T)$ as temperaturedependent self-energy. The equilibrium distribution $n(T)$ is a particular solution of an effective kinetic theory of BoltzmannVlasov type [96] with force $F^{\alpha}(x)=\left[\partial^{\alpha} \Pi(x)\right] /[2 \sqrt{\Pi(x)}]$ [71,72], satisfying $p_{\alpha} F^{\alpha}=0$ for the momentum $p^{\alpha} \sim$ $(E(\vec{p}, x), \vec{p})$, in a state without entropy production where the collision term vanishes. Thermodynamic self-consistency is ensured, i.e., $\epsilon(T) \equiv T_{(0)}^{\mu v} u_{\mu} u_{v}, P(T) \equiv-\frac{1}{3} T_{(0)}^{\mu \nu} \Delta_{\mu \nu}$, and $\epsilon=T^{2} \partial[P(T) / T] / \partial T$ from $\epsilon+P=T s$ with $s=\partial P / \partial T$.

Equation (1) for the shear viscosity, which was first derived in the quasiparticle framework in [101], coincides formally with the results $[102,103]$ obtained within kinetic theory without the force term $\propto F^{\alpha}$ and without self-energy. Nonetheless, it differs from these results as the effective quasiparticle mass $\Pi(T)$ entering $E$ reflects medium effects. This was also observed in $[60,61,104,105]$.

The expression (2) for the bulk viscosity represents a special case of the general form of $\zeta, \frac{1}{T} \int \frac{d^{3} \vec{p}}{(2 \pi)^{3}} n(T)[1+$ $\left.\left.d^{-1} n(T)\right] \frac{\tau}{E^{2}}\left\{\left[E^{2}-a\right] \frac{\partial P}{\partial \epsilon}-\frac{1}{3} \vec{p}^{2}\right\} X\left[E^{2}-a\right]-\frac{1}{3} \vec{p}^{2}\right\}$, obtained by setting the momentum-independent factor $X=\partial P / \partial \epsilon$ for invoking the Landau-Lifshitz condition. (Choosing instead $\quad X=\frac{1}{3}, \quad \frac{1}{T} \int \frac{d^{3} \vec{p}}{(2 \pi)^{3}} n(T)\left[1+d^{-1} n(T)\right] \frac{\tau}{E^{2}} \frac{1}{3}\left\{\left[E^{2}-\right.\right.$ a] $\left.\frac{\partial P}{\partial \epsilon}-\frac{1}{3} \vec{p}^{2}\right\}\{\Pi(T)-a\}$ would follow for $\zeta$, which corrects a factor-of-2 mistake in the result derived in [101].) Equation (2) differs even formally from the previous results $[60,61,102-105]$. This is because it contains the subtracted mass term $[\Pi(T)-a]$ as in $[72,77,101]$ and thus also the derivative of $G^{2}(T)$ with respect to $T$ and not only $\Pi(T)$ alone. If one would use a temperature-independent mass squared $M^{2}$ instead of $\Pi(T)$ in $E$, then both results for $\zeta$ would correspond to the expression obtained in [103].

Medium (i.e., EoS) effects influence the bulk viscosity more prominently than the shear viscosity. This is because $\zeta$, just opposite to $\eta$, is relevant in processes that change the volume of a system rather than its shape. Then, in the pure gluon plasma, the only way to relax locally the system to equilibrium is by changing the average energy of the excitations and, thus, the energy density.

Discussing the general properties of $\zeta$ from Eq. (2), we note that, for a gas of particles with small and constant, i.e., temperature-independent, mass, a bulk viscosity $\zeta \simeq 0$ is found in the ultrarelativistic limit $\left(p^{\alpha} p_{\alpha} \simeq 0\right)$ because $\partial P / \partial \epsilon \simeq 1 / 3$. In the nonrelativistic case, $T_{(1) i}^{i} \equiv 2 T_{(1)}^{00}$ is obtained from the above decomposition of the energy-momentum tensor, which in the local fluid rest frame vanishes due to the Landau-Lifshitz condition implying also $\zeta=0$. For massless particles, one has exactly $\partial P / \partial \epsilon=1 / 3, \partial \Pi / \partial T=0$, and $E=|\vec{p}|$, such that $\zeta=0$ is found from Eq. (2) in line with $[98,100,102,103,106]$.
In the conformal limit, i.e., at asymptotically large $T$, the bulk viscosity vanishes $\zeta \rightarrow 0$. This is because the squared factor in Eq. (2) can be rewritten as

$$
\begin{aligned}
{\left[E^{2}-a\right] \frac{\partial P}{\partial \epsilon}-\frac{1}{3} \vec{p}^{2} } & =\vec{p}^{2}\left(\frac{\partial P}{\partial \epsilon}-\frac{1}{3}\right)+\frac{\partial P}{\partial \epsilon}[\Pi(T)-a] \\
& =\vec{p}^{2}\left(\frac{\partial P}{\partial \epsilon}-\frac{1}{3}\right)-\frac{1}{2} \frac{\partial P}{\partial \epsilon} T^{4} \frac{\partial G^{2}}{\partial T^{2}}
\end{aligned}
$$

for $\Pi(T)=T^{2} G^{2}(T) / 2$ (cf. Sec. II C). Consequently, in the combination $[\Pi(T)-a]$, the leading temperature dependence of $\Pi(T)$ is canceled out (cf. also [77]), leaving solely the derivative of the effective coupling with respect to $T$. In the conformal limit, however, the only dimensionful scale is the temperature. This prohibits, in particular, a running of the coupling with $T$, i.e., $\partial G^{2} / \partial T=0$. Therefore, together with $\partial P / \partial \epsilon=1 / 3$ [cf. Eq. (12) below], one finds

$$
\vec{p}^{2}\left(\frac{\partial P}{\partial \epsilon}-\frac{1}{3}\right)-\frac{1}{2} \frac{\partial P}{\partial \epsilon} T^{4} \frac{\partial G^{2}}{\partial T^{2}}=0 .
$$

In other words, the terms $(\partial P / \partial \epsilon-1 / 3)$ and $[\Pi(T)-a]$ are a measure for the deviation of the system from conformal invariance.

\section{B. Relaxation times}

To quantify shear and bulk viscosities from Eqs. (1) and (2), respectively, one needs to specify $\tau$. Microscopically considered, the shear viscosity is dominantly determined by elastic two-body scatterings among excitations at typical momenta of order $\mathcal{O}(T)$. The bulk viscosity, in contrast, measures relaxation of disturbances, which are caused by a slow uniform expansion of the system. Consequently, it is determined by inelastic particle number changing processes and is sensitive to excitations with soft momenta $[71,72,75-77,98]$. This implies that the relaxation times for $\eta$ and $\zeta$ are, in general, different. In the following, however, we concentrate on elastic gluon-gluon $2 \leftrightarrow 2$ scatterings only. The corresponding relaxation rate $\tau^{-1}=\tilde{n}\langle\tilde{v} \sigma\rangle$ is determined from the thermal-averaged total (elastic) scattering cross section $\langle\tilde{v} \sigma\rangle$ and the particle density in local equilibrium $\tilde{n}$, where $\tilde{v}$ is the relative velocity between the two scattering particles.

The expression we employ as ansatz for $\tau^{-1}$ originates from solving the Boltzmann equation by a variational treatment, including screening effects in the gluon-gluon scattering [107]. The result depends crucially on the ratio of maximumto-minimum momentum transfer in the scattering process. The minimum momentum transfer is related to the Debye screening mass $m_{D}$. The maximum momentum transfer $p_{\max }$ is instead limited by the typical particle momentum in the medium. For our quasiparticles, this is $4>p_{\max } / T>3$ in the temperature interval $1<T / T_{c}<4$, while for larger $T$, $p_{\max } / T$ decreases extremely slowly with $T$ and can be set constant.

At large temperatures, the relaxation rate $\tau^{-1}$ was found to be proportional to $\ln \left(1 / m_{D}^{2}\right)$ [107]. In order to extend this perturbative QCD result into the nonperturbative regime, we replace the running coupling $g^{2}=4 \pi \alpha_{s}$ entering $m_{D}$ by 
the effective coupling $G^{2}(T)$ from Eq. (5) below. In this way, one arrives at the following phenomenological form for the inverse relaxation time:

$$
\tau^{-1}=a_{1} T G^{4} \ln \left(a_{2} / G^{2}\right) .
$$

The above expression is momentum independent and is assumed to be common for shear and bulk viscosities. This may be considered as a rather crude approximation [75,77,108]. A more refined approach, employing a momentum-dependent relaxation time including elastic and inelastic scatterings, was recently proposed in [95].

\section{Temperature-dependent self-energy}

For the quantitative discussion of Eqs. (1) and (2), one also has to specify the self-energy $\Pi(T)$. We adopt the quasiparticle model [86-91] with $\Pi(T)=T^{2} G^{2}(T) / 2$ and use the effective coupling [86-89]

$$
G^{2}(T)=\frac{16 \pi^{2}}{\left[11 \ln \left(\frac{T-T_{s}}{T_{c} / \lambda}\right)^{2}\right]}
$$

inspired by the QCD one-loop running coupling for purely gluonic matter. In fact, by construction, the effective coupling $G^{2}(T)$ reproduces $g^{2}(T)$ at large $T$, where a temperature dependence is introduced into $g^{2}$ by considering it at variable renormalization point $2 \pi T c$ with $c \in[1 / 2,2]$, and as scale $T_{c} / \Lambda_{\overline{M S}}=1.14$ from $[109,110]$ is used. This implies, in particular, that $G^{2}(T)$ vanishes logarithmically as $T \rightarrow \infty$. In Eq. (5), $\lambda$ and $T_{s}$ are two model parameters.

This quasiparticle model works impressively well in describing lattice QCD results for the equation of state and related quantities by adjusting $\lambda$ and $T_{s}$. Figure 1 exhibits the comparison of QPM results for the scaled interaction measure $(\epsilon-3 P) / T^{4}$ as a function of $T / T_{c}$ with the corresponding lattice QCD results obtained in pure $\mathrm{SU}_{c}(3)$ gauge theory $[111,112]$. The model parameters were chosen as $\lambda=4.3$ and $T_{s}=0.73 T_{c}$, with $T_{c}=271 \mathrm{MeV}$ [113]. In order to obtain $\epsilon$

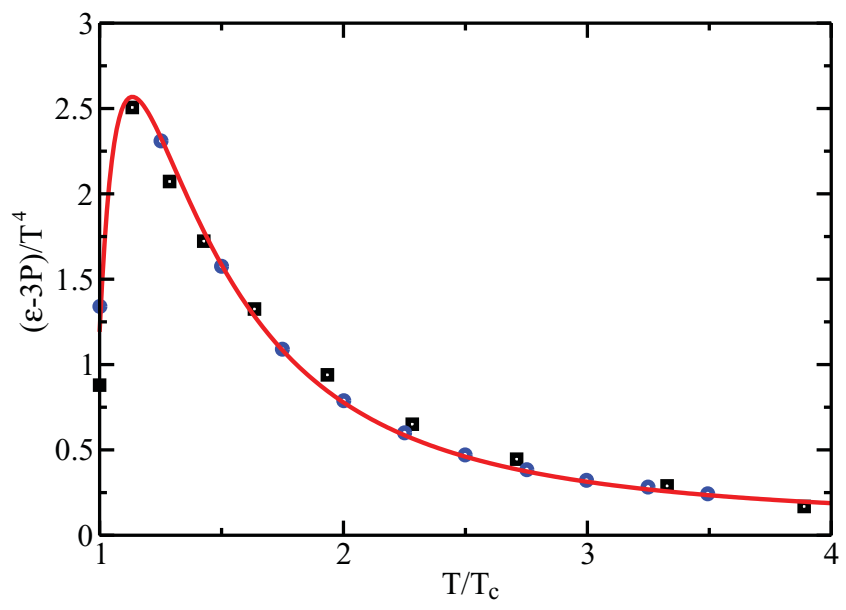

FIG. 1. (Color online) Comparison of QPM results (solid curve) for the scaled interaction measure $(\epsilon-3 P) / T^{4}$ as a function of $T / T_{c}$ for the pure gluon plasma with $\mathrm{SU}_{c}(3)$ lattice $\mathrm{QCD}$ results from [111] (boxes) and from [112] (circles). and $P[91,92]$, we have evaluated

$$
\begin{aligned}
\epsilon(T) & =\int \frac{d^{3} \vec{p}}{(2 \pi)^{3}} \operatorname{En}(T)+B(T), \\
P(T) & =\int \frac{d^{3} \vec{p}}{(2 \pi)^{3} E} \frac{\vec{p}^{2}}{3} n(T)-B(T),
\end{aligned}
$$

where $B(T)$ is the solution of $\partial B(T) / \partial \Pi(T)=-\frac{1}{2}$ $\int \frac{d^{3} \vec{p}}{(2 \pi)^{3} E} n(T)$, with integration constant $B\left(T_{c}\right)=0.19 T_{c}^{4}$. Thereby, it is implicitly assumed that $B(T)$ depends on $T$ only via $\Pi(T)$. As evident from Fig. 1, both the maximum in the vicinity of $T_{c}$, which signals the strong deviation of $\mathrm{SU}_{c}$ (3) Yang-Mills theory from the conformal limit $\epsilon=3 P$ [114], as well as the decline toward the conformal limit for asymptotically large $T$ observed in lattice QCD are well described within the QPM. The latter behavior follows from the logarithmic decrease of the effective coupling $G^{2}(T)$ with $T$, while the position of the maximum is related to the inflection point of $p / T^{4}$ as a function of $\ln T$ and depends on the choices made for $\lambda$ and $T_{s}$.

We note as an aside that the presence of dynamical quarks significantly influences the thermodynamics of the system (cf., e.g., [115] for corresponding lattice QCD calculations using physical quark masses). Similarly, it is expected that also the transport coefficients are considerably different for QCD compared to the pure gluon plasma. It would be interesting to study how these differences eventually translate into the specific viscosity coefficients.

The squared speed of sound as a function of $T / T_{c}$ following the QPM result for the interaction measure exhibited in Fig. 1 can be parametrized in the temperature interval $T_{c}<T \leqslant 4 T_{c}$ by

$$
\frac{\partial P}{\partial \epsilon}=\frac{1}{3} \tanh \left[\sum_{n=1}^{5} c_{n}\left(T / T_{c}-1\right)^{n}\right],
$$

where the parameters read as $c_{1}=4.78, c_{2}=-6.39, c_{3}=$ $4.60, c_{4}=-1.57$, and $c_{5}=0.20$. The quality of this fit is quantified by $\chi^{2} /$ d.o.f. $=3.89 \times 10^{-4} / 16$. The function $\partial P / \partial \epsilon$ is monotonously rising with $T$, from small values just above $T_{c}$ toward $1 / 3$; at $T=4 T_{c}$, the squared speed of sound is only $2.6 \%$ smaller than $1 / 3$. At $T \rightarrow T_{c}^{+}$, it decreases quickly to zero as expected for a first-order phase transition [116].

The high-temperature expansion of $P(T)$ from Eq. (7) for the pure $\mathrm{SU}_{c}(3)$ plasma

$$
P(T)=a T^{4}\left[1-b G^{2}(T)+\mathcal{O}\left(G^{4}\right)\right],
$$

with $a=d \pi^{2} / 90$ and $b=15 /\left(d \pi^{2}\right)$, was shown in [87] to be in agreement with the pQCD result [77]. The squared speed of sound at large $T$ can be evaluated from standard thermodynamic relations $\partial P / \partial \epsilon=d P / d \epsilon=(d P / d T) /(d \epsilon / d T)=$ $(d P / d T) /\left(T d^{2} P / d T^{2}\right)$, where the individual derivatives 
read as

$$
\frac{d P}{d T}=4 a T^{3}\left[1-b G^{2}+\mathcal{O}\left(G^{4}\right)\right]-a b T^{4} \frac{d G^{2}}{d T}+\mathcal{O}\left(d G^{4} / d T\right),
$$$$
\begin{aligned}
T \frac{d^{2} P}{d T^{2}}= & 12 a T^{3}\left[1-b G^{2}+\mathcal{O}\left(G^{4}\right)\right]-8 a b T^{4} \frac{d G^{2}}{d T} \\
& +\mathcal{O}\left(d^{2} G^{2} / d T^{2}, d G^{4} / d T\right)
\end{aligned}
$$

From Eq. (5) and at large $T$, the following pattern is valid: $1 \gg G^{2} \gg T\left(d G^{2} / d T\right) \gg T^{2}\left(d^{2} G^{2} / d T^{2}\right)$. Thus, the squared speed of sound in the gluon plasma at large temperatures is given by

$$
\frac{\partial P}{\partial \epsilon}=\frac{1}{3}+\frac{5}{36} b T \frac{d G^{2}}{d T}+\mathcal{O}\left(G^{2} T d G^{2} / d T\right) .
$$

The temperature derivative of the effective coupling follows from Eq. (5) as

$$
\left(T-T_{s}\right) \frac{d G^{2}}{d T}=-\frac{11}{8 \pi^{2}} G^{4}(T) .
$$

Therefore, for large $T \gg T_{s}$, one finds $T\left(d G^{2} / d T\right)=$ $-11 G^{4}(T) /\left(8 \pi^{2}\right)<0$. In other words, $\partial P / \partial \epsilon \leqslant 1 / 3$ is obtained from Eq. (12). For asymptotically large $T, \partial P / \partial \epsilon$ approaches $1 / 3$.

\section{THE SHEAR VISCOSITY}

In [76], the shear viscosity was obtained for the pure gluon plasma with $N_{c}=3$ colors at next-to-leading log order of small running coupling $g$ as

$$
\eta_{\mathrm{NLL}}=\frac{\eta_{1} T^{3}}{g^{4} \ln \left[\mu_{*} /(g T)\right]}
$$

with $\eta_{1}=27.126$ and $\mu_{*} / T=2.765$. With $\tau$ from Eq. (4) and at large $T$, the expression for $\eta$ in Eq. (1) exhibits parametrically the same dependencies on coupling and on temperature as the above perturbative result. Thus, within our formalism, by choosing the parameters in Eq. (4) appropriately, one can adjust the QPM to reproduce the above pQCD result well and then extrapolate the latter into the region close to $T_{c}$.

Taking into account the behavior of $G^{2}(T)$ for large $T$, we choose $a_{2}=\left(\mu_{*} / T\right)^{2}$ in order to recover the constant in the logarithm of $\eta_{\mathrm{NLL}}$. Furthermore, the QPM expression for the shear viscosity from Eq. (1) can be written as $\eta=T^{4} b(T) \tau$. Here, $b(T)$ is a dimensionless and with $T$ monotonically rising function. It approaches slowly its asymptotic limit $b(T \rightarrow$ $\infty)=d \Gamma(4) \zeta(4) /\left(30 \pi^{2}\right) \simeq 1.404$ following the logarithmic decrease of $G^{2}(T) \rightarrow 0$ as $T \rightarrow \infty$. Thus, by setting $a_{1} \simeq$ $2.587 \times 10^{-2}$, our $\eta \rightarrow \eta_{\mathrm{NLL}}$ for $T \rightarrow \infty$. Variations in $a_{1}$, keeping $a_{2}$ fixed, allow us to consider cases where $\eta \rightarrow \eta_{\mathrm{NLL}}$ for a large but finite temperature.

The quantitative behavior of the shear viscosity to entropy density ratio $\eta / s$ in the QPM as a function of $T / T_{c}$ is shown in Fig. 2 for different values of $a_{1}$ and $a_{2}$. The dashed curve corresponds to $a_{1} \simeq 2.587 \times 10^{-2}$ and $a_{2}=\left(\mu_{*} / T\right)^{2}$ (Fit 1$)$, while the solid curve corresponds to $a_{1}=3.85 \times 10^{-2}$ and

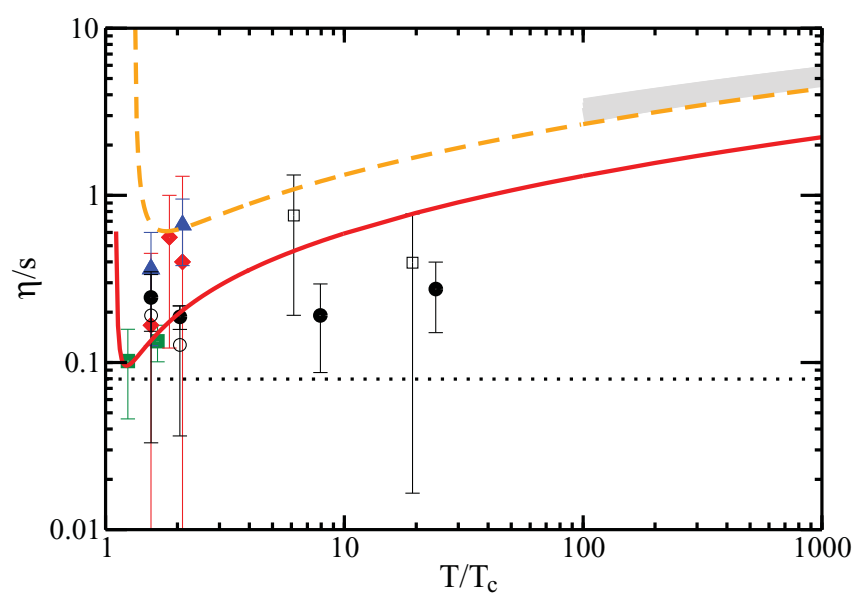

FIG. 2. (Color online) Specific shear viscosity $\eta / s$ as a function of $T / T_{c}$. Dashed and solid curves exhibit the QPM results for Fit 1 and Fit 2, respectively (see text for details). The symbols denote lattice QCD data for pure $\mathrm{SU}_{c}$ (3) gauge theory from [64] (full squares), from [66] (diamonds and triangles), and from [67] (open squares, open and full circles). The dotted line exhibits the Kovtun-Son-Starinets bound [45-47] $(\eta / s)_{\mathrm{KSS}}=1 /(4 \pi)$. The gray band at large $T$ indicates the perturbative QCD result $\eta_{\mathrm{NLL}} / \tilde{s}$ with $\eta_{\mathrm{NLL}}$ from [76] and entropy density $\tilde{s}$ from [117]. The band was obtained by varying the renormalization point $\bar{\mu}$ in the QCD running coupling $g(\bar{\mu})$ in the range $\pi T \leqslant \bar{\mu} \leqslant 4 \pi T$.

$a_{2}=2\left(\mu_{*} / T\right)^{2}$ (Fit 2). The entropy density $s$ in the QPM (cf. $[91,92])$ was calculated by using the parametrization (5) for $G^{2}(T)$, entering also the viscosities, such that results on equilibrium thermodynamics for the pure gluon plasma obtained in lattice QCD are reproduced (see Fig. 1). The model results are compared with perturbative QCD results and with recent lattice QCD data in Fig. 2.

As evident from Fig. 2, the ratio $\eta / s$ is very sensitive to the values of $a_{1}$ and $a_{2}$ in Eq. (4). However, irrespective of the particular choices made for these parameters, the specific shear viscosity calculated from Eq. (1) decreases with decreasing $T$ and exhibits a minimum at $T \gtrsim T_{c}$. The precise location of this minimum is determined by the value of $a_{2}$. For even smaller $T$, $\eta / s$ increases again monotonically as $T \rightarrow T_{c}^{+}$. This picture is qualitatively in agreement with model predictions for the confined phase reporting a monotonically decreasing $\eta / s$ for $T \rightarrow T_{c}^{-}$[118-120]. For both fits, the ratio $\eta / s$ is found to be significantly smaller than unity in a temperature region around its minimum, which is in contrast to naive extrapolations of pure pQCD results.

With the parameters from Fit 1 , the specific shear viscosity approaches for asymptotically large $T$ the result expected from perturbative calculations (see Fig. 2). At smaller temperatures close to $T_{c}$, agreement with some, but not the general bulk of, available lattice QCD data $[64,66,67]$ is found. Exhibiting a minimum of $(\eta / s)_{\min } \simeq 0.6$ at $T \simeq 1.75 T_{c}, \eta / s$ rises to quite large values for $T \rightarrow T_{c}^{+}$. This, however, seems to be unfavored by recent results obtained for $T \lesssim T_{c}$ in the confined phase [118-120].

Clearly, the extraction of transport coefficients from lattice QCD simulations is a difficult task $[59,79]$ and results seem far 


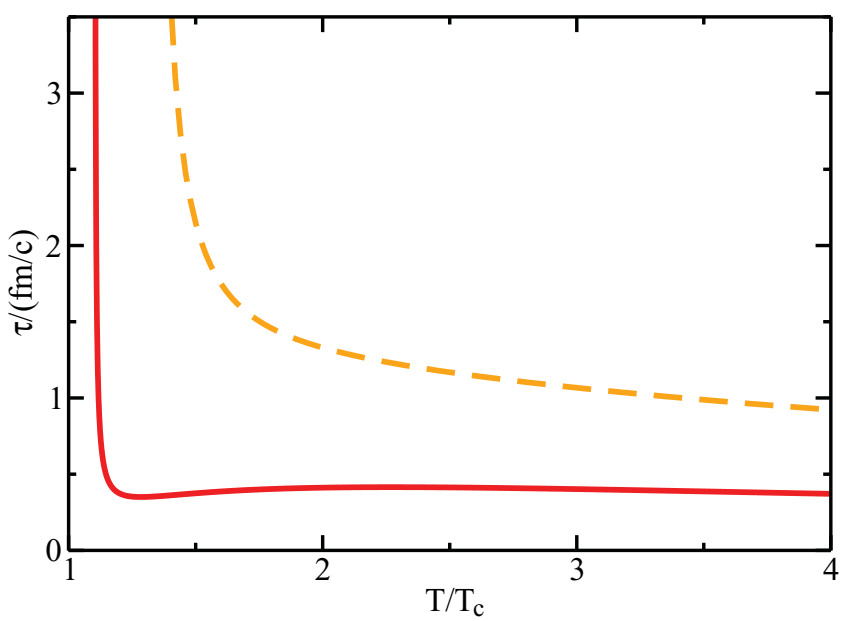

FIG. 3. (Color online) Relaxation time $\tau$ from Eq. (4) for the parameters from Fit 1 (dashed curve) and Fit 2 (solid curve). See text for details.

from being conclusive. Following the arguments in [121,122], we focus on the lattice QCD results for $\eta / s$ from [64]. These results are best described with the parameters from Fit 2. In addition, as evident from Fig. 2, this parametrization shows fairly nice agreement with available lattice QCD results over a wide range of temperatures (see also [101]).

The behavior of $\eta / s$ observed in Fig. 2 is a direct consequence of the particular form of $\tau$. In Fig. 3, the relaxation time is exhibited as a function of $T / T_{C}$ for the different parameter sets corresponding to Fits 1 and 2 . While being rather flat at moderate temperatures above $T_{c}, \tau$ increases rapidly for decreasing temperatures. In particular, in the case of Fit 2, a pronounced rise of $\tau$ in the vicinity of $T_{c}$ is observed. This behavior is in line with the phenomenon of critical slowing down, indicating that the amount of time needed for relaxing energy and momentum density fluctuations increases rapidly near $T_{c}$.

Interestingly, the sharp increase of $\tau$ close to $T_{c}$ is driven by the logarithm in Eq. (4). Without the logarithm, i.e., when assuming that $\tau$ depends solely on $\left(T G^{4}\right)^{-1}$, the relaxation time would simply decrease with decreasing $T$ in line with the increase of $G^{2}(T)$ for $T \rightarrow T_{c}^{+}$according to Eq. (5). However, close to $T_{c}$, the factor $\ln \left(a_{2} / G^{2}\right)$ becomes dominant and determines the behavior of $\tau$. The precise temperature dependence is crucially driven by the parameter $a_{2}$, which can lead to potentially huge quantitative differences [108]. Increasing (decreasing) $a_{2}$ results in a shift of the sharp increase in $\tau$ to smaller (larger) $T$, apart from an overall decrease (increase) of $\tau$.

As evident from Eq. (4), the relaxation time diverges at $T=T^{*}$ for which $G^{2}\left(T^{*}\right) \equiv a_{2}$. For the parametrization of $G^{2}(T)$ employed in Fig. 1, we find $T^{*} \simeq 1.32 T_{c}$ (Fit 1) and $T^{*} \simeq 1.1 T_{c}$ (Fit 2). For $T<T^{*}$, the relaxation time in Eq. (4) becomes negative, indicating that the used ansatz is not valid anymore.

At large $T$, the behavior of $\tau$ is dominated by its $(1 / T)$ dependence such that $\tau \rightarrow 0$ for $T \rightarrow \infty$. However, in $\eta=$ $T^{4} b(T) \tau$, this temperature dependence of $\tau$ is compensated.
The two different parametrizations Fit 1 and Fit 2 of $\tau$ result in a finite difference between the corresponding shear viscosities denoted by $\eta^{(1)}$ for Fit 1 (using $a_{1}^{(1)}$ and $a_{2}^{(1)}$ ) and $\eta^{(2)}$ for Fit 2 (using $a_{1}^{(2)}$ and $a_{2}^{(2)}$ ). At large $T$, the ratio $\eta^{(1)} / \eta^{(2)}$ reads as

$$
\frac{\eta^{(1)}}{\eta^{(2)}}=\frac{a_{1}^{(2)}}{a_{1}^{(1)}}\left(1+\frac{\ln 2}{\ln \left[\left(\mu^{*} / T\right)^{2} / G^{2}\right]}\right) \rightarrow \frac{a_{1}^{(2)}}{a_{1}^{(1)}} \simeq 1.49 .
$$

Therefore, by construction, the ratio $\eta^{(1)} / s$ approaches the pQCD result for asymptotically large $T$, whereas $\eta^{(2)} / s$ does not reach this limit at any $T$, remaining at least a factor $a_{1}^{(1)} / a_{1}^{(2)}$ smaller.

With the parameters from Fit 2, we find for the relaxation time $\tau \simeq 0.4 \mathrm{fm} / \mathrm{c}$ for $T \geqslant 1.2 T_{c}$, whereas it increases sharply beyond $5 \mathrm{fm} / \mathrm{c}$ as $T \rightarrow T_{c}^{+}$(see Fig. 3). Similar values were used in the hydrodynamic simulations [38] for discussing the influence of shear and bulk viscosities on observables measured in heavy-ion collisions such as the elliptic flow. Moreover, our results for $\tau$ are numerically comparable with results obtained in [78,123], where a different ansatz for $\tau$ based on [124,125] was used, reading as

$$
\tau^{-1}=\frac{2 N_{c}}{8 \pi} T G^{2} \ln \left(\frac{2 c}{G^{2}}\right) .
$$

The parameter $c$ is determined by demanding $\tau \rightarrow \infty$ as $T \rightarrow T_{c}$, i.e., $c \equiv G^{2}\left(T=T_{c}\right) / 2$. The results for $\eta / s$ from $[78,104,105]$, where this ansatz for $\tau$ is employed, show a slightly flatter $T$ dependence compared with our Fit 2 . However, Eq. (14) differs from Eq. (4) in its parametric dependence on $G^{2}$. Therefore, as already discussed in [78], the parametric dependence of $\eta$ on the coupling found in pQCD can not be recovered with Eq. (14) for $\tau^{-1}$.

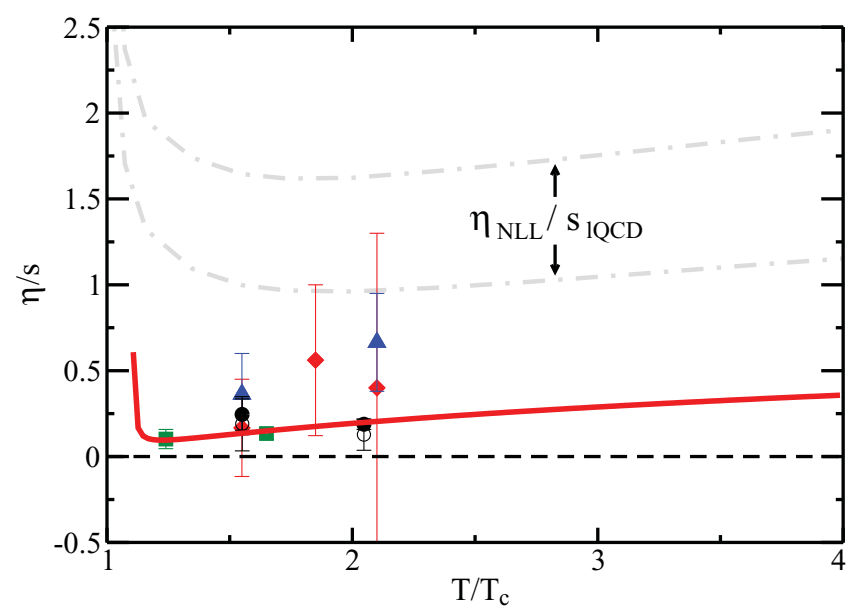

FIG. 4. (Color online) Specific shear viscosity $\eta / s$ as a function of $T / T_{c}$ as in Fig. 2 but zoomed into the temperature region near $T_{c}$. Symbols depict lattice QCD results as in Fig. 2 and the solid curve exhibits the QPM result for Fit 2. The area between the two gray dashed-dotted curves shows the extrapolation of the $\mathrm{pQCD}$ result $\eta_{\mathrm{NLL}}$ from [76] scaled by the entropy density from lattice QCD [111,112], where the renormalization point entering $\eta_{\mathrm{NLL}}$ is varied between $\bar{\mu}=$ $\pi T$ and $\bar{\mu}=4 \pi T$. 
As evident from Fig. 4, the ratio $\eta / s$ for Fit 2 increases very mildly with increasing $T$. For $T=1.5 T_{c}$, we find $\eta / s=0.13$, which is a factor $1 / 3$ smaller than results obtained in [126] for the quark-gluon plasma with $N_{f}=3$ degenerate quark flavors. At $T=3 T_{c}, \eta / s \simeq 0.29$ is still small and only a factor of 3 larger than the minimum value $(\eta / s)_{\min }=0.096$. A similar value for the minimum was recently reported from a virial expansion approach [127].

\section{THE BULK VISCOSITY}

The bulk viscosity exhibits different parametric dependencies on $T$ and on the coupling in comparison with the shear viscosity as observed in pQCD calculations [75-77]. In the QPM at large $T$, one finds with the expression for the squared speed of sound Eq. (12) that Eq. (3), which enters $\zeta$ quadratically, exhibits for thermal quasiparticle momenta the following structure:

$$
\begin{aligned}
& \vec{p}^{2}\left(\frac{\partial P}{\partial \epsilon}-\frac{1}{3}\right)-\frac{1}{2} \frac{\partial P}{\partial \epsilon} T^{4} \frac{\partial G^{2}}{\partial T^{2}} \\
& =\frac{5 b}{36} T \frac{\partial G^{2}}{\partial T} \vec{p}^{2}-\frac{1}{12} T^{3} \frac{\partial G^{2}}{\partial T}+\mathcal{O}\left(G^{6}\right) \sim T^{2} G^{4}(T) .
\end{aligned}
$$

Together with $\tau^{-1}$ from Eq. (4), this implies for the bulk viscosity at large $T$, where $E \simeq|\vec{p}| \sim T$, that

$$
\zeta \sim \frac{T^{3} G^{4}(T)}{\ln \left[a_{2} / G^{2}(T)\right]} .
$$

Keeping in mind the behavior of $G^{2}(T)$ at large $T$, this result coincides with the leading-log-order parametric behavior of $\zeta$ obtained in pQCD calculations by an expansion in inverse powers of $\ln \left(1 / \alpha_{s}\right)$ [77]. Nonetheless, our result for $\zeta$ exhibits the parametric dependencies on $T$ and $G^{2}(T)$ in Eq. (16) only, because it contains the subtracted mass term $[\Pi(T)-a]$ rather than the effective thermal mass $\Pi(T)$ of the quasiparticle excitations alone.

The QPM results for the specific bulk viscosity as a function of $T / T_{c}$ are depicted for Fit 2 in Fig. 5 and compared with available lattice QCD results $[65,67]$. We find a positive but approximately vanishing ratio $\zeta / s$ for $T \geqslant 2 T_{c}$ in line with lattice QCD. However, for $T \rightarrow T_{c}^{+}$, the specific bulk viscosity increases sharply following the behavior of $\tau$. Similarly, for $T \rightarrow T_{c}^{-}$in the confined phase, a monotonic increase of $\zeta / s$ was found in different model calculations [118,129-131]. Both observations together suggest that, for strongly interacting matter, the ratio $\zeta / s$ develops a maximum in the vicinity of $T_{c}$ [55-58]. Such a behavior is, for instance, also seen in Lennard-Jones fluids [132].

Even though the QPM results are in qualitative agreement with lattice QCD, nonetheless, for $T<2 T_{c}$, they are systematically below the lattice QCD data [65]. However, the results from [65] are accompanied by noticeable uncertainties and a less rapid increase of $\zeta / s$ close to $T_{c}$ seems to be likely [122]. In fact, refined calculations [122] have been reported for the combined specific sound channel $(\eta+3 \zeta / 4) / s$, however, only for temperatures, where the contribution from $\eta$ is dominant.

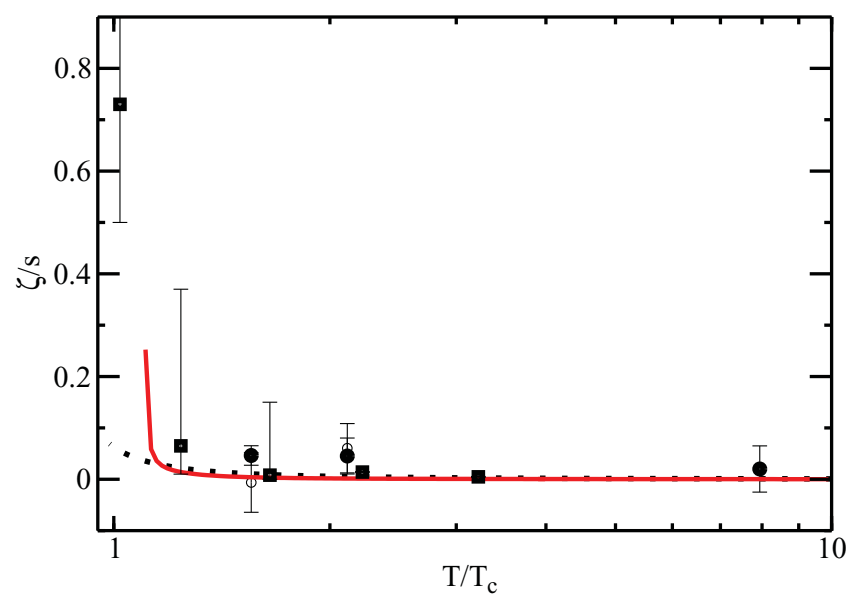

FIG. 5. (Color online) Specific bulk viscosity $\zeta / s$ as a function of $T / T_{c}$. The solid curve exhibits the QPM result for Fit 2. It is compared with available lattice QCD data from [65] (full squares) and [67] (open and full circles). The noticeable error bars for the results from [65] represent conservative upper and lower bounds given in [65]. The dotted curve shows, for comparison, results recently reported from holographic QCD [128].

A comparison of QPM predictions for the specific sound channel with available lattice QCD data from [122] and from a combination of data from $[64,65]$ is exhibited in Fig. 6, showing fairly nice agreement.

Our results for $\zeta / s$ and $\eta / s$ exhibit both a pronounced behavior close to $T_{c}$, which might result in a sizable influence of these transport coefficients on some observables measured in heavy-ion collisions. In fact, the observed possible rapid increase of $\eta / s$ with decreasing $T$ close to $T_{c}$ as well as the behavior with $T$ in the confined phase [118-120] call for refinements in viscous hydrodynamic simulations [133-135].

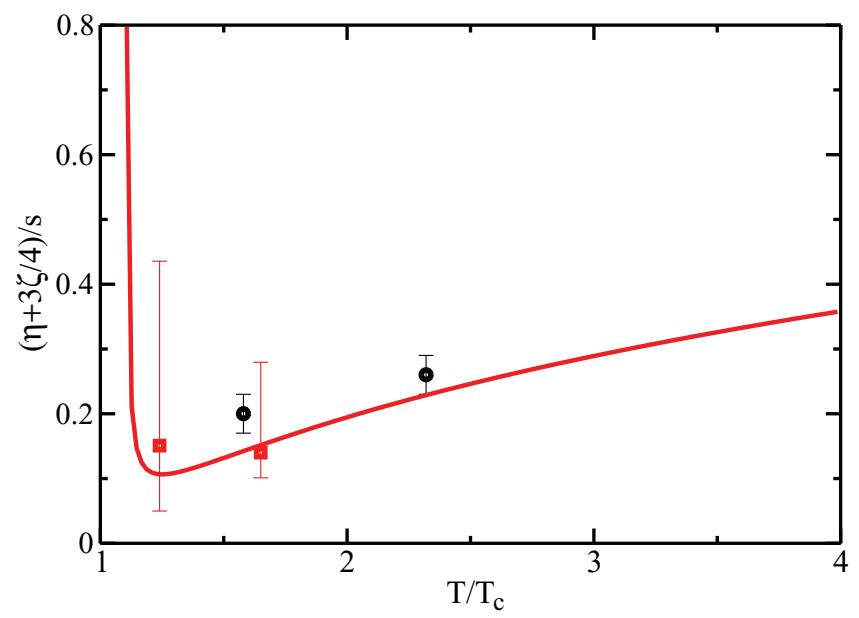

FIG. 6. (Color online) Combined specific sound channel $(\eta+$ $3 \zeta / 4) / s$ as a function of $T / T_{c}$. The QPM result for Fit 2 is shown by the solid curve. It is compared with available lattice QCD results taken either directly from [122] (circles) or from combining results of [64] and [65] (squares). The uncertainties in the latter arise from the upper and lower bounds in $\zeta / s$ depicted in Fig. 5. 
The smallness of $\zeta / s$ over a wide range of $T$, in contrast, could suggest that the impact of bulk viscous effects is less important. Indeed, in [38] a small influence of $\zeta / s$ on the shear viscous suppression of the elliptic flow was found. Although $\zeta / s$ becomes larger close to $T_{c}$, it increases less than the corresponding relaxation time (see Figs. 3 and 5) due to the thermodynamic integrals, cf. Eq. (2). In other words, critical slowing down hampers the influence of bulk viscosity as long as the corresponding (negative) bulk viscous pressure is small enough to ensure the stability conditions of viscous hydrodynamics [38]. This effect also tempers the tendency of the fluid to become mechanically unstable against cavitation and clustering for large values of $\zeta[37,136-138]$.

\section{CONCLUSIONS}

Shear and bulk viscosities give important information about the transport properties of a medium. In this paper, we calculated these transport coefficients for the pure gluon plasma within a quasiparticle model by assuming the plasma to be describable in terms of (quasi)gluon excitations with temperature-dependent self-energy.

The approach is based on an effective Boltzmann-Vlasovtype kinetic theory for quasiparticle excitations with mediumdependent dispersion relation, which is consistent with the approach proposed in $[71,72]$. In local thermal equilibrium, this picture reduces to the quasiparticle model, which was employed successfully to describe lattice QCD equilibrium thermodynamics in [90,92-94]. Thermodynamic self-consistency in this model is a direct consequence of the consistency condition that must be imposed in order to fulfill energy and momentum conservation in the kinetic theory [95,101]. We used expressions for shear and bulk viscosities derived within relaxation-time approximation to the kinetic theory. In the shear viscosity, medium effects appear only implicitly via the dispersion relation. In the bulk viscosity, medium effects are, in addition, explicitly reflected by the subtracted mass term, which contains the derivative of the effective coupling with respect to the temperature.

Including only contributions from elastic gluon-gluon scatterings to the relaxation time, we have shown that, at large $T$, our results for the transport coefficients exhibit the same parametric dependencies on temperature and coupling as found in pQCD calculations [75-77]. For this to hold true, it is crucial that the bulk viscosity contains the subtracted mass term rather than the self-energy alone.

In the temperature region close to $T_{c}$, fairly nice quantitative agreement with available lattice QCD results for the transport coefficients is found. The specific shear viscosity $\eta / s$ exhibits a minimum in the vicinity of $T_{c}$, while the specific bulk viscosity $\zeta / s$, being large close to $T_{c}$, falls off rapidly to its conformal limit for larger $T$.

For the specific shear viscosity, we find a rather mild increase with temperature. In fact, the ratio $\eta / s$ is smaller than unity even at temperatures being a few times larger than $T_{c}$, e.g., at $T \simeq 3 T_{c}$, it is still about 0.3 . Even though our investigations were limited to the case of a pure gluon plasma, one might expect that for thermal conditions relevant in heavyion collisions at the LHC (Large Hadron Collider), the ratio $\eta / s$ is small such that predictions for the LHC heavy-ion program based on ideal hydrodynamic simulations are meaningful $[92,139,140]$. However, to make definite predictions, it would be of importance to include also quark degrees of freedom into our quasiparticle approach.

\section{ACKNOWLEDGMENTS}

We gratefully acknowledge valuable and insightful discussions with P. Braun-Munzinger, B. Friman, S. Jeon, H. B. Meyer, S. Peigné, A. Peshier, C. Sasaki, and U. Wiedemann. We also thank J. Kapusta for expressing his opinion on this paper. The work was supported by BMBF 06 DR 9059, GSI-FE, the European Network I3-HP2 Toric, and the Polish Ministry of Science.
[1] J. Adams et al. (STAR Collaboration), Nucl. Phys. A 757, 102 (2005).

[2] K. Adcox et al. (PHENIX Collaboration), Nucl. Phys. A 757, 184 (2005).

[3] I. Arsene et al. (BRAHMS Collaboration), Nucl. Phys. A 757, 1 (2005).

[4] B. B. Back et al. (PHOBOS Collaboration), Nucl. Phys. A 757, 28 (2005)

[5] P. F. Kolb, P. Huovinen, U. Heinz, and H. Heiselberg, Phys. Lett. B 500, 232 (2001).

[6] P. Huovinen, P. F. Kolb, U. W. Heinz, P. V. Ruuskanen, and S. A. Voloshin, Phys. Lett. B 503, 58 (2001).

[7] D. Teaney, J. Lauret, and E. V. Shuryak, Phys. Rev. Lett. 86, 4783 (2001).

[8] T. Hirano and K. Tsuda, Phys. Rev. C 66, 054905 (2002).

[9] P. F. Kolb and R. Rapp, Phys. Rev. C 67, 044903 (2003).
[10] P. F. Kolb and U. Heinz, in Quark Gluon Plasma 3, edited by R. C. Hwa and X. N. Wang (World Scientific, Singapore, 2004), p. 634.

[11] M. Miller and R. Snellings, arXiv:nucl-ex/0312008.

[12] T. Hirano, U. W. Heinz, D. Kharzeev, R. Lacey, and Y. Nara, Phys. Lett. B 636, 299 (2006).

[13] M. Luzum and P. Romatschke, Phys. Rev. C 78, 034915 (2008); 79, 039903(E) (2009).

[14] H. Petersen, J. Steinheimer, G. Burau, and M. Bleicher, Eur. Phys. J. C 62, 31 (2009).

[15] Y. Hama, R. P. G. Andrade, F. Grassi, W.-L. Qian, and T. Kodama, Acta Phys. Pol. B 40, 931 (2009).

[16] K. Werner, T. Hirano, I. Karpenko, T. Pierog, S. Porteboeuf, M. Bleicher, and S. Haussler, J. Phys. G: Nucl. Part. Phys. 36, 064030 (2009).

[17] H. Song and U. W. Heinz, Nucl. Phys. A 830, 467C (2009).

[18] T. Hirano and Y. Nara, Nucl. Phys. A 830, 191C (2009). 
[19] U. Heinz and P. F. Kolb, Nucl. Phys. A 702, 269 (2002).

[20] R. A. Lacey, N. N. Ajitanand, J. M. Alexander, P. Chung, W. G. Holzmann, M. Issah, A. Taranenko, P. Danielewicz, and H. Stöcker, Phys. Rev. Lett. 98, 092301 (2007).

[21] H.-J. Drescher, A. Dumitru, C. Gombeaud, and J.-Y. Ollitrault, Phys. Rev. C 76, 024905 (2007).

[22] M. Gyulassy and L. D. McLerran, Nucl. Phys. A 750, 30 (2005).

[23] E. V. Shuryak, Nucl. Phys. A 750, 64 (2005).

[24] U. W. Heinz, arXiv:nucl-th/0512051.

[25] A. Muronga, Phys. Rev. Lett. 88, 062302 (2002); 89, 159901(E) (2002).

[26] D. Teaney, Phys. Rev. C 68, 034913 (2003).

[27] A. Muronga and D. H. Rischke, arXiv:nucl-th/0407114.

[28] U. W. Heinz, H. Song, and A. K. Chaudhuri, Phys. Rev. C 73, 034904 (2006).

[29] R. Baier, P. Romatschke, and U. A. Wiedemann, Phys. Rev. C 73, 064903 (2006)

[30] A. K. Chaudhuri, Phys. Rev. C 74, 044904 (2006).

[31] P. Romatschke and U. Romatschke, Phys. Rev. Lett. 99, 172301 (2007).

[32] A. Muronga, Phys. Rev. C 76, 014909 (2007).

[33] A. K. Chaudhuri, arXiv:0708.1252; arXiv:0801.3180.

[34] K. Dusling and D. Teaney, Phys. Rev. C 77, 034905 (2008).

[35] D. Molnar and P. Huovinen, J. Phys. G: Nucl. Part. Phys. 35, 104125 (2008).

[36] H. Song and U. W. Heinz, Phys. Lett. B 658, 279 (2008); Phys. Rev. C 77, 064901 (2008); 78, 024902 (2008).

[37] R. J. Fries, B. Müller, and A. Schäfer, Phys. Rev. C 78, 034913 (2008).

[38] H. Song and U. W. Heinz, Phys. Rev. C 81, 024905 (2010).

[39] W. Israel and J. M. Stewart, Ann. Phys. (NY) 118, 341 (1979).

[40] W. A. Hiscock and L. Lindblom, Ann. Phys. (NY) 151, 466 (1983).

[41] R. Baier, P. Romatschke, D. T. Son, A. O. Starinets, and M. A. Stephanov, J. High Energy Phys. 04, 100 (2008).

[42] B. Betz, D. Henkel, and D. H. Rischke, Prog. Part. Nucl. Phys. 62, 556 (2009).

[43] M. A. York and G. D. Moore, Phys. Rev. D 79, 054011 (2009).

[44] P. Danielewicz and M. Gyulassy, Phys. Rev. D 31, 53 (1985).

[45] G. Policastro, D. T. Son, and A. O. Starinets, Phys. Rev. Lett. 87, 081601 (2001).

[46] P. Kovtun, D. T. Son, and A. O. Starinets, J. High Energy Phys. 10, 064 (2003).

[47] P. K. Kovtun, D. T. Son, and A. O. Starinets, Phys. Rev. Lett. 94, 111601 (2005).

[48] S. Gavin and M. Abdel-Aziz, Phys. Rev. Lett. 97, 162302 (2006).

[49] Y. Kats and P. Petrov, J. High Energy Phys. 01, 044 (2009).

[50] T. D. Cohen, Phys. Rev. Lett. 99, 021602 (2007).

[51] A. Cherman, T. D. Cohen, and P. M. Hohler, J. High Energy Phys. 02, 026 (2008).

[52] A. Jakovac, Phys. Rev. D 81, 045020 (2010).

[53] L. P. Csernai, J. I. Kapusta, and L. D. McLerran, Phys. Rev. Lett. 97, 152303 (2006)

[54] T. Schäfer, Phys. Rev. A 76, 063618 (2007).

[55] D. Kharzeev and K. Tuchin, J. High Energy Phys. 09, 093 (2008).

[56] F. Karsch, D. Kharzeev, and K. Tuchin, Phys. Lett. B 663, 217 (2008).

[57] P. Romatschke and D. T. Son, Phys. Rev. D 80, 065021 (2009).

[58] K. Paech and S. Pratt, Phys. Rev. C 74, 014901 (2006).
[59] G. D. Moore and O. Saremi, J. High Energy Physics 09, 015 (2008).

[60] C. Sasaki and K. Redlich, Phys. Rev. C 79, 055207 (2009).

[61] C. Sasaki and K. Redlich, Nucl. Phys. A 832, 62 (2010).

[62] A. Buchel, Phys. Lett. B 663, 286 (2008).

[63] A. Buchel, Phys. Lett. B 681, 200 (2009).

[64] H. B. Meyer, Phys. Rev. D 76, 101701 (2007).

[65] H. B. Meyer, Phys. Rev. Lett. 100, 162001 (2008).

[66] A. Nakamura and S. Sakai, Phys. Rev. Lett. 94, 072305 (2005).

[67] S. Sakai and A. Nakamura, PoS LAT2007, 221 (2007).

[68] R. Kubo, J. Phys. Soc. Jpn. 12, 570 (1957).

[69] A. Hosoya, M. Sakagami, and M. Takao, Ann. Phys. (NY) 154, 229 (1984).

[70] T. Schäfer and D. Teaney, Rep. Prog. Phys. 72, 126001 (2009).

[71] S. Jeon, Phys. Rev. D 52, 3591 (1995).

[72] S. Jeon and L. G. Yaffe, Phys. Rev. D 53, 5799 (1996).

[73] J. S. Gagnon and S. Jeon, Phys. Rev. D 75, 025014 (2007); 76, 089902 (2007).

[74] J. S. Gagnon and S. Jeon, Phys. Rev. D 76, 105019 (2007).

[75] P. Arnold, G. D. Moore, and L. G. Yaffe, J. High Energy Phys. 11, 001 (2000).

[76] P. Arnold, G. D. Moore, and L. G. Yaffe, J. High Energy Phys. 05, 051 (2003).

[77] P. Arnold, C. Dogan, and G. D. Moore, Phys. Rev. D 74, 085021 (2006)

[78] A. Peshier and W. Cassing, Phys. Rev. Lett. 94, 172301 (2005).

[79] G. Aarts and J. M. Martinez Resco, J. High Energy Phys. 04, 053 (2002).

[80] G. Aarts and J. M. Martinez Resco, J. High Energy Phys. 02, 061 (2004).

[81] Z. Xu and C. Greiner, Phys. Rev. C 71, 064901 (2005).

[82] Z. Xu and C. Greiner, Phys. Rev. Lett. 100, 172301 (2008).

[83] A. El, A. Muronga, Z. Xu, and C. Greiner, Phys. Rev. C 79, 044914 (2009).

[84] J.-W. Chen, H. Dong, K. Ohnishi, and Q. Wang, Phys. Lett. B 685, 277 (2010).

[85] J.-W. Chen, J. Deng, H. Dong, and Q. Wang, Phys. Rev. D 83, 034031 (2011).

[86] A. Peshier, B. Kämpfer, O. P. Pavlenko, and G. Soff, Phys. Lett. B 337, 235 (1994).

[87] A. Peshier, B. Kämpfer, O. P. Pavlenko, and G. Soff, Phys. Rev. D 54, 2399 (1996).

[88] A. Peshier, B. Kämpfer, and G. Soff, Phys. Rev. C 61, 045203 (2000).

[89] A. Peshier, B. Kämpfer, and G. Soff, Phys. Rev. D 66, 094003 (2002).

[90] M. Bluhm, B. Kämpfer, and G. Soff, Phys. Lett. B 620, 131 (2005).

[91] M. Bluhm, B. Kämpfer, R. Schulze, and D. Seipt, Eur. Phys. J. C 49, 205 (2007).

[92] M. Bluhm, B. Kämpfer, R. Schulze, D. Seipt, and U. Heinz, Phys. Rev. C 76, 034901 (2007).

[93] M. Bluhm and B. Kämpfer, Phys. Rev. D 77, 034004 (2008).

[94] M. Bluhm and B. Kämpfer, Phys. Rev. D 77, 114016 (2008).

[95] P. Chakraborty and J. I. Kapusta, Phys. Rev. C 83, 014906 (2011).

[96] S. R. de Groot, W. A. van Leeuwen, and C. G. van Weert, Relativistic Kinetic Theory (North-Holland, Amsterdam, 1980).

[97] L. D. Landau and E. M. Lifshitz, Fluid Mechanics, 2nd ed. (Butterworth and Heinemann, London, 1987). 
[98] E. M. Lifshitz and L. P. Pitaevskii, Physical Kinetics (Pergamon, Oxford, 1981).

[99] S. Weinberg, Gravitation and Cosmology (Wiley, New York, 1972).

[100] S. Weinberg, Astrophys. J. 168, 175 (1971).

[101] M. Bluhm, B. Kämpfer, and K. Redlich, Nucl. Phys. A 830, 737C (2009).

[102] A. Hosoya and K. Kajantie, Nucl. Phys. B 250, 666 (1985).

[103] S. Gavin, Nucl. Phys. A 435, 826 (1985).

[104] A. S. Khvorostukhin, V. D. Toneev, and D. N. Voskresensky, Nucl. Phys. A 845, 106 (2010).

[105] A. S. Khvorostukhin, V. D. Toneev, and D. N. Voskresensky, Phys. Rev. C 83, 035204 (2011).

[106] R. Horsley and W. Schoenmaker, Nucl. Phys. B 280, 716 (1987).

[107] H. Heiselberg, Phys. Rev. D 49, 4739 (1994).

[108] U. Heinz, Proceedings of Banff/CAP Workshop on Thermal Field Theories (World Scientific, Singapore, 1994).

[109] O. Kaczmarek, F. Karsch, F. Zantow, and P. Petreczky, Phys. Rev. D 70, 074505 (2004).

[110] O. Kaczmarek and F. Zantow, Phys. Rev. D 71, 114510 (2005).

[111] G. Boyd, J. Engels, F. Karsch, E. Laermann, C. Legeland, M. Lutgemeier, and B. Petersson, Nucl. Phys. B 469, 419 (1996).

[112] M. Okamoto et al., Phys. Rev. D 60, 094510 (1999).

[113] F. Karsch, Lect. Notes Phys. 583, 209 (2002).

[114] E. Megias, E. R. Arriola, and L. L. Salcedo, Phys. Rev. D 80, 056005 (2009).

[115] S. Borsanyi, G. Endrodi, Z. Fodor, A. Jakovac, S. D. Katz, S. Krieg, C. Ratti, and K. K. Szabo, J. High Energy Phys. 11, 077 (2010).

[116] Z. Fodor and S. D. Katz, arXiv:0908.3341.

[117] J.-P. Blaizot, E. Iancu, and A. Rebhan, Phys. Rev. D 63, 065003 (2001).

[118] J. Noronha-Hostler, J. Noronha, and C. Greiner, Phys. Rev. Lett. 103, 172302 (2009).
[119] D. Fernandez-Fraile and A. Gomez Nicola, Eur. Phys. J. C 62 , 37 (2009).

[120] J.-W. Chen and E. Nakano, Phys. Lett. B 647, 371 (2007).

[121] H. B. Meyer, J. High Energy Phys. 08, 031 (2008).

[122] H. B. Meyer, Nucl. Phys. A 830, 641C (2009).

[123] A. Peshier, Phys. Rev. D 70, 034016 (2004).

[124] R. D. Pisarski, Phys. Rev. Lett. 63, 1129 (1989).

[125] V. C. Lebedev and A. V. Smilga, Ann. Phys. (NY) 202, 229 (1990).

[126] B. A. Gelman, E. V. Shuryak, and I. Zahed, Phys. Rev. C 74, 044908 (2006).

[127] S. Mattiello and W. Cassing, Eur. Phys. J. C 70, 243 (2010)

[128] U. Gürsoy, E. Kiritsis, G. Michalogiorgakis, and F. Nitti, J. High Energy Phys. 12, 056 (2009).

[129] D. Fernandez-Fraile and A. Gomez Nicola, Phys. Rev. Lett. 102, 121601 (2009).

[130] A. Wiranata and M. Prakash, Nucl. Phys. A 830, 219C (2009).

[131] J.-W. Chen and J. Wang, Phys. Rev. C 79, 044913 (2009).

[132] K. Meier, A. Laesecke, and S. Kabelac, J. Chem. Phys. 122, 014513 (2005).

[133] N. Demir and S. A. Bass, Phys. Rev. Lett. 102, 172302 (2009).

[134] G. S. Denicol, T. Kodama, and T. Koide, J. Phys. G: Nucl. Part. Phys. 37, 094040 (2010).

[135] P. Bozek, Phys. Rev. C 81, 034909 (2010).

[136] G. Torrieri and I. Mishustin, Phys. Rev. C 78, 021901 (2008).

[137] G. Torrieri, B. Tomasik, and I. Mishustin, Phys. Rev. C 77, 034903 (2008).

[138] K. Rajagopal and N. Tripuraneni, J. High Energy Phys. 03, 018 (2010).

[139] N. Armesto et al., J. Phys. G: Nucl. Part. Phys. 35, 054001 (2008).

[140] K. Aamodt et al. (ALICE Collaboration), arXiv:1011.3914. 